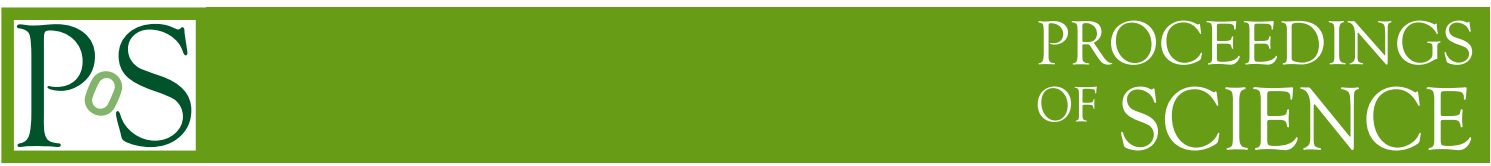

\title{
Observing event horizons with high-frequency VLBI
}

\section{Vincent Fish ${ }^{* \dagger}$}

MIT Haystack Observatory, USA

E-mail: vfish@haystack.mit.edu

Due to recent technological and scientific advancements, VLBI at $1.3 \mathrm{~mm}$ wavelength has matured to a point where observations can readily achieve an angular resolution better than $60 \mathrm{mi}-$ croarcseconds. Using millimetre VLBI arrays, our group has embarked on a project of observing the black holes at the centre of our Galaxy (the 4 million $M_{\odot}$ Sagittarius A*) and the giant elliptical galaxy M87 on event horizon scales. Our data are consistent with the $1.3 \mathrm{~mm}$ emission in Sgr A* coming from a very compact region offset from the black hole, as predicted by general relativistic effects. Within the context of physically-motivated models, our long-baseline detections can be used to place constraints on the orientation and spin of the black hole in Sgr A*. A series of observations in April 2009 revealed an increase in the flux density of the compact emitting region in Sgr A* on a timescale of less than a day while the size of the emitting region remained constant. In M87, the size of the emission region obtained from high-frequency VLBI places constraints on the high-energy emission region and may be important for discriminating among models of jet genesis near a black hole. Future improvements to the (sub)millimetre VLBI array will result in a true Event Horizon Telescope Horizon Telescope capable of producing transformative science.

10th European VLBI Network Symposium and EVN Users Meeting: VLBI and the new generation of radio arrays

September 20-24, 2010

Manchester, $U K$

*Speaker.

${ }^{\dagger}$ on behalf of the Event Horizon Telescope collaboration 


\section{Introduction}

Very high angular resolution is the key to understanding a variety of complex astrophysical phenomena. Among these is the accretion and outflow region surrounding black holes. A large variety of theoretical models for the emission region exist, since "single-pixel" observations are presently unable to distinguish whether a disk or an outflow is responsible for the (sub)millimetre emission in the Sagittarius A*, the radio source associated with the massive black hole at the centre of the Galaxy. Sgr A* may be an excellent source for testing general relativity in a strong-gravity environment, but the accretion and outflow astrophysics, which dominate the electromagnetic radiation, must be understood first.

Of all known black hole candidates, Sgr A* has the largest apparent event horizon as viewed from the Earth, with a Schwarzschild radius of approximately $10 \mu$ as. The black hole at the centre of the nearby galaxy M87 has a Schwarzschild radius that is nearly as large. Millimetre-wavelength VLBI has the requisite resolution to observe these scales, with angular resolutions as small as $30 \mu$ as at $230 \mathrm{GHz}$ on Hawaii-Chile and Europe-Chile baselines (and $20 \mu$ as at $345 \mathrm{GHz}$ ). For Sgr A* especially, high-frequency observations are necessary due to interstellar scattering, which dominates over the intrinsic source size at $\lambda=3 \mathrm{~mm}$ and longer [i]. The intrinsic size of the emitting region is itself wavelength-dependent due to opacity effects, with smaller sizes at shorter wavelength (higher frequency). Short millimetre observations $(\lambda \leq 1.3 \mathrm{~mm})$ are therefore necessary to probe the inner accretion/outflow region of $\mathrm{Sgr} \mathrm{A}^{*}$.

\section{Observations of $\mathrm{Sgr} \mathrm{A}^{*}$ in $\mathbf{2 0 0 7}$}

In 2007, we observed $\mathrm{Sgr} \mathrm{A}^{*}$ at $230 \mathrm{GHz}$ using a three-telescope array consisting of the James Clerk Maxwell Telescope (JCMT) in Hawaii, the Submillimeter Telescope (SMT) in Arizona, and one telescope of the Combined Array for Research in Millimeter-wave Astronomy (CARMA) in California. The lengths of these baselines range from about $900 \mathrm{~km}$ (SMT-CARMA) to over $4000 \mathrm{~km}$ (JCMT-CARMA and JCMT-SMT). We successfully obtained fringes on the SMT-CARMA and JCMT-SMT baselines [1]]. The fringes on the latter baseline (with a fringe spacing $\sim 60 \mu$ as) conclusively demonstrate the existence of structure on spatial scales of a few Schwarzschild radii.

Due to the relative sparseness of detections in the $(u, v)$ plane, we considered two simple models of the emitting region of Sgr A*: a circular Gaussian and a uniform ring (Figure 1). Physically, a circular Gaussian might correspond approximately to a one-dimensional cut across the intensity profile of an inclined disk, where Doppler boosting causes the approaching side of the disk to dominate the emission [2]. Likewise, a uniform ring might approximate the emission from a face-on disk, where the central portion of the disk is not visible due to the "shadow" created by the black hole [3]. Both models could fit the 2007 data for physically reasonable model parameters.

The circular Gaussian model of the emission yields a size of $43 \mu$ as, or $37 \mu$ as $\left(3.7 \mathrm{R}_{\mathrm{Sch}}\right.$ ) after the effects of interstellar scattering are removed. This very small size rules out the possibility that the $230 \mathrm{GHz}$ emission is optically thick and centred on the black hole, since gravitational lensing would cause such emission to appear to have a diameter of 5.2 $\mathrm{R}_{\mathrm{Sch}}$ for a Schwarzschild black hole 


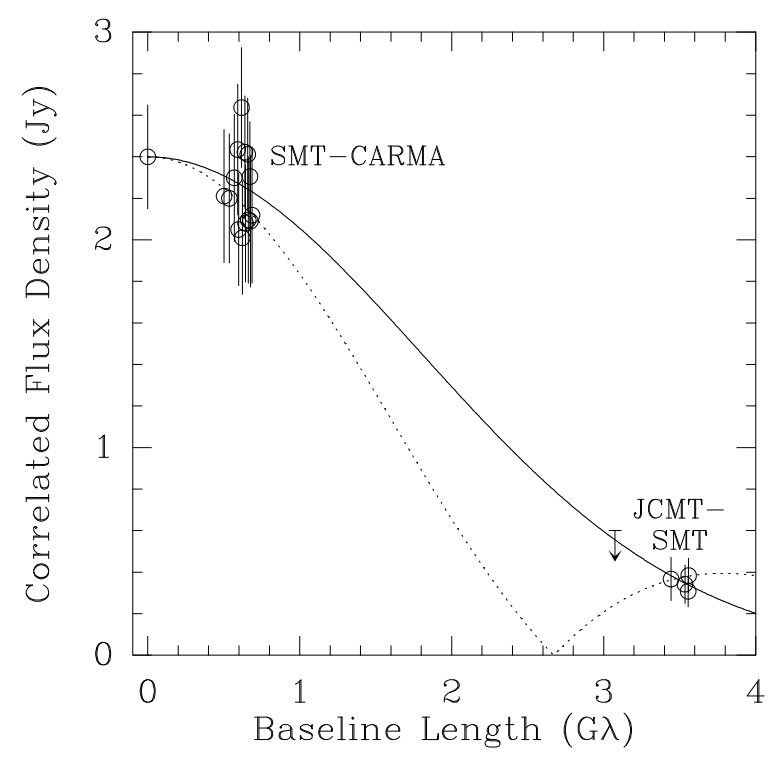

Figure 1: Millimetre VLBI detections of Sgr A* in 2007 [1]. The arrow near $3 \mathrm{G} \lambda$ denotes the upper limit from nondetections on the JCMT-CARMA baseline. The solid line shows the best Gaussian fit to the data, implying a size of $43 \mu$ as. The dotted line shows a possible fit assuming the emission comes from a uniform ring instead.

[1]. The very compact size of the emitting region also strengthens the case that $\operatorname{Sgr} \mathrm{A} *$ has an event horizon [凹].

An alternate approach to model fitting is to generate a library of models based on prior physical assumptions (e.g., jets, general relativisitic magnetohydrodynamic (GRMHD) flows, etc.) parameterized by unknowns about the Sgr A* system (e.g., the three-dimensional spin vector of the black hole and the accretion rate of the system) and then compare the data against model visibility predictions. For instance, if the emission around $\mathrm{Sgr} \mathrm{A}^{*}$ can be approximated by a radiatively inefficient

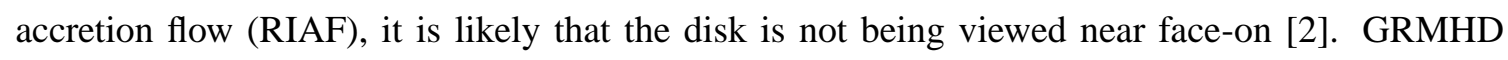
models reach a similar conclusion about the disk inclination [5, 6]. These types of analysis can also be useful in planning future observations [7, 87 .

\section{Observations of $\operatorname{Sgr} A^{*}$ in 2009}

In April 2009, we observed Sgr A* with a four-telescope array consisting of the JCMT, SMT, and two CARMA telescopes operated independently (with separate VLBI backends) [9]. Sgr A* was detected on all baselines (Figure 2), including numerous detections on the long baselines to Hawaii. Three features of the 2009 data point toward new results.

First, unlike in 2007, the flux density on the SMT-CARMA VLBI baseline is clearly lower than the "zero-spacing" flux measured by both the ultrashort CARMA-CARMA VLBI baseline and contemporaneous CARMA interferometer measurements. (Baselines shorter than $20 \mathrm{k} \lambda$ are excluded to remove contributions from dust in the greater Galactic Centre region.) The $(u, v)$ coverage of the VLBI detections is still sufficiently sparse to permit multiple models of the source structure to 

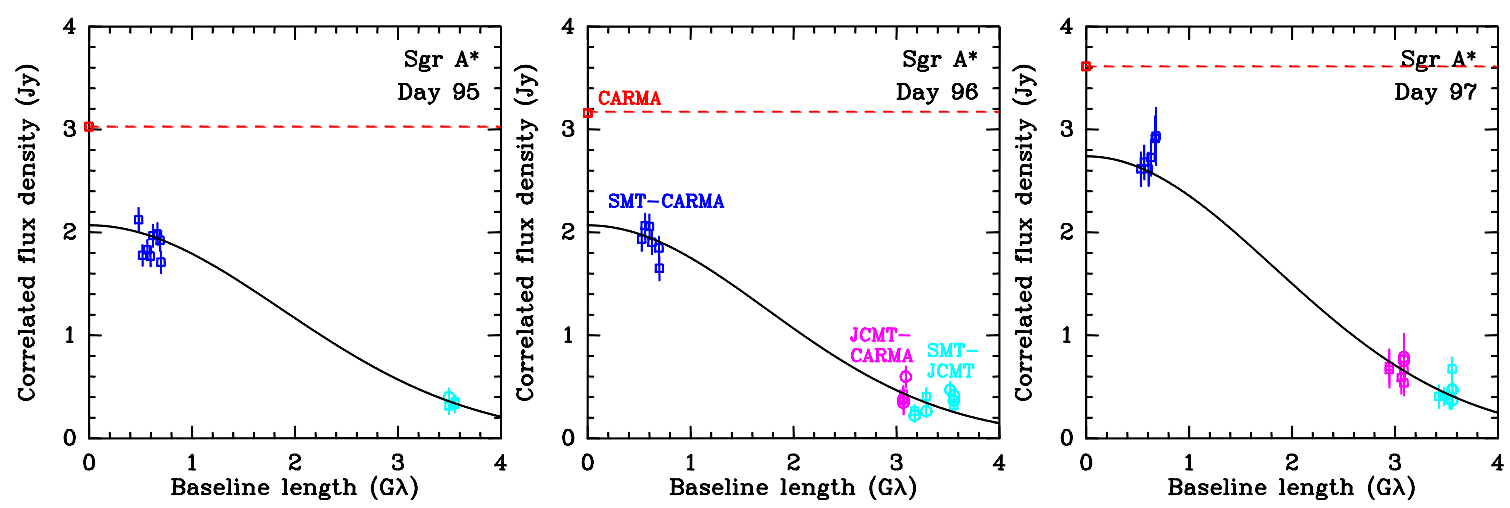

Figure 2: Observations of Sgr A* in April 2009 [9]. The red point and dashed line indicate the best estimate of the average total flux density of Sgr A* on each day. The dark blue points show detections on the short SMT-CARMA baselines. The magenta and cyan points show detections on the long JCMT-CARMA and JCMT-SMT baselines, respectively. The black line shows the best Gaussian fit to the data on each day. In each case, the size is consistent with $43 \mu$ as.

fit the data. On the assumption that the total emission consists of a compact component and an extended component, the latter not contributing significantly to the correlated SMT-CARMA flux densities, the lower limit on the size of the extended component is approximately $300 \mu$ as. The flux density in the extended component must be slowly variable on long timescales, since the total flux density is much higher than in 2007.

Second, the correlated flux density on the JCMT-CARMA baseline generally exceeds that of the longer JCMT-SMT baseline. Whilst it is still possible to fit the compact emission with a ring model when the inner radius is treated as a free parameter, general relativity predicts that the apparent size of the inner radius of an accretion disk should depend only on the ratio of the mass of Sgr A* to its distance from us, which is known to an accuracy of a few percent [10], thus placing a strong constraint on the expected location of a null in correlated visibility amplitude plots. In a wide variety of physically realistic families of accretion flow models, low-inclination disk models are disfavoured by the JCMT-CARMA detections [2, 5, 11, 12].

Third, the flux density of the compact component is seen to be larger on the third night of observations in 2009 compared to the first two nights, but its size remains constant. Treating the 2007 data and each of the three days of observations in 2009 as four independent data sets, the best-fit circular Gaussian is always consistent with having a FWHM size of $43 \mu$ as [9]. Sgr A* appears to be in a new steady state on the third night of observations, with a higher flux density but similar size as compared to the previous nights. Observations at $\lambda=3$ and $7 \mathrm{~mm}$ are inconclusive as to whether a significant correlation exists between flux density and source size [13, 14] but, due to the increased opacity of the accretion flow, are only sensitive to the accretion flow at several tens of Schwarzschild radii from the black hole.

Detections of Sgr A* on the longest baselines (JCMT-CARMA and JCMT-SMT) occur over a time span of 3 hours on one night of observing, which is long enough for the baseline tracks to trace out substantial arcs in $(u, v)$ space. Future observations and analyses may be able to identify whether the source exhibits measurable deviations from axisymmetry [12]. 


\section{M87}

The nearby galaxy M87 is also detected on very long baselines at $1.3 \mathrm{~mm}$. As at $7 \mathrm{~mm}$, the $1.3 \mathrm{~mm}$ VLBI data detects both the large-scale jet and a compact component [15, 16, 17]. Highresolution observations such as those provided by millimetre-wavelength VLBI may be necessary to place strong constraints on parameters of the jet, such as the size of the jet footprint, its collimation, and the spin of the black hole in M87 [18].

\section{Future Directions}

Several technological advancements will permit future observations to benefit from higher sensitivity, which will be necessary for probing time-variable structural changes in Sgr A* 19 , [20]. A phased-array processor has been developed to sum the collecting area from up to eight telescopes on Mauna Kea (the JCMT, the Caltech Submillimeter Observatory, and telescopes of the Submillimeter Array), and a similar instrument will be deployed at CARMA [21]. Current observations have been recorded at $4 \mathrm{Gbit} \mathrm{s}^{-1}$, and wider-bandwidth observations in the near future will double or quadruple the bit rate. New telescopes will also be incorporated into the observing array, producing an Event Horizon Telescope with significantly enhanced sensitivity and $(u, v)$ coverage.

\section{Summary}

Millimetre VLBI observations of Sgr A* in 2007 and 2009 have demonstrated the existence of structure on scales of a few Schwarzschild radii, furthering the scientific case that Sgr A* contains a black hole with an event horizon. Multiple geometrical models can fit the millimetre VLBI data, but most of the millimetre emission appears to originate in a compact component whose size was not seen to change in the aftermath of a brightening of the source in 2009. In the context of physically-motivated models of the accretion region, our data suggest that the spin vector of the black hole is inclined to the line of sight with the accretion disk being viewed more than $30^{\circ}$ from face-on. Long-baseline detections of M87 will enable similar studies of the compact jet launch region around a different class of supermassive black holes. Observations in the near future will have significantly greater sensitivity and better $(u, v)$ coverage, enabling detailed studies of the quiescent and variable accretion flow in Sgr $\mathrm{A}^{*}$.

\section{Acknowledgments}

High-frequency VLBI work at MIT Haystack Observatory is supported by grants from the National Science Foundation.

\section{References}

[1] S. S. Doeleman et al. 2008, Event-horizon-scale structure in the supermassive black hole candidate at the Galactic Centre, Nature 45578. 
[2] A. E. Broderick, V. L. Fish, S. S. Doeleman, \& A. Loeb 2009, Estimating the Parameters of Sagittarius A*'s Accretion Flow via Millimeter VLBI, ApJ 69745.

[3] H. Falcke, F. Melia, \& E. Agol 2000, Viewing the Shadow of the Black Hole at the Galactic Center, ApJ 528 L13.

[4] A. E. Broderick, A. Loeb, \& R. Narayan 2009, The Event Horizon of Sagittarius A*, ApJ 7011357.

[5] M. Mościbrodzka, C. F. Gammie, J. C. Dolence, H. Shiokawa, \& P. K. Leung 2009, Radiative Models of Sgr A* from GRMHD Simulations, ApJ 706497.

[6] J. Dexter, E. Agol, P. C. Fragile, \& J. C. McKinney 2010, The Submillimeter Bump in Sgr A*from Relativistic MHD Simulations, ApJ 7171092.

[7] V. L. Fish, A. E. Broderick, S. S. Doeleman, \& A. Loeb 2009, Using Millimeter VLBI to Constrain RIAF Models of Sagittarius A*, ApJ 692 L14.

[8] L. Huang, R. Takahashi, \& Z.-Q. Shen 2009, Testing the Accretion Flow with Plasma Wave Heating Mechanism for Sagittarius A* by the $1.3 \mathrm{~mm}$ VLBI Measurements, ApJ, 706960.

[9] V. L. Fish et al. 2010, $1.3 \mathrm{~mm}$ Wavelength VLBI of Sagittarius A*: Detection of Time-Variable Emission on Event Horizon Scales, ApJL, submitted, arXiv:1011.2472

[10] A. M. Ghez et al. 2008, Measuring Distance and Properties of the Milky Way's Central Supermassive Black Hole with Stellar Orbits, ApJ 6891044.

[11] J. Dexter. E. Agol, \& P. C. Fragile 2009, Millimeter Flares and VLBI Visibilities from Relativistic Simulations of Magnetized Accretion onto the Galactic Center Black Hole, ApJ 703 L142

[12] A. E. Broderick, V. L. Fish, S. S. Doeleman, \& A. Loeb 2010, Evidence for Low Black Hole Spin and Physically Motivated Accretion Models from Millimeter VLBI Observations of Sagittarius A*, ApJ, submitted, arXiv:1011.2770

[13] G. C. Bower, H. Falcke, R. M. Herrnstein, J.-H. Zhao, W. M. Goss, \& D. C. Backer 2004, Detection of the Intrinsic Size of Sagittarius A* Through Closure Amplitude Imaging, Science 304704.

[14] R.-S. Lu, T. P. Krichbaum, A. Eckart, S. König, D. Kunneriath, G. Witzel, A. Witzel, \& J. A. Zensus 2010, Multiwavelength VLBI observations of Sagittarius A*, $A \& A$, in press, arXiv:1010.1287

[15] C. Ly, R. C. Walker, \& W. Junor 2007, High-Frequency VLBI Imaging of the Jet Base of M87, ApJ 660200.

[16] V. A. Acciari et al. 2009, Radio Imaging of the Very-High-Energy $\gamma$-Ray Emission Region in the Central Engine of a Radio Galaxy, Science 325444.

[17] S. S. Doeleman et al., in preparation.

[18] A. E. Broderick \& A. Loeb 2009, Imaging the Black Hole Silhouette of M87: Implications for Jet Formation and Black Hole Spin, ApJ 6971164.

[19] S. S. Doeleman, V. L. Fish, A. E. Broderick, A. Loeb, \& A. E. E. Rogers 2009, Detecting Flaring Structures in Sagittarius A* with High-Frequency VLBI, ApJ 69559.

[20] V. L. Fish, S. S. Doeleman, A. E. Broderick, A. Loeb, \& A. E. E. Rogers 2009, Detecting Changing Polarization Structures in Sagittarius A* with High Frequency VLBI, ApJ 7061353.

[21] J. Weintroub 2008, A submillimeter VLBI array, J. Phys. Conf. Ser. P131 012047 